\title{
Elaboração e análise sensorial de doce de mamão verde com coco e pimenta ${ }^{1}$
}

\author{
Priscila Duarte Silva ${ }^{2}$, Elizanilda Ramalho do Rêgo ${ }^{3}$, Angela Maria dos Santos Pessoa ${ }^{3}$, Nardiele de \\ Souza Souto Freitas ${ }^{2}$, Manoel Ricardo de Andrade Júnior ${ }^{3}$, Maílson Monteiro do Rêgo ${ }^{3}$
}

\begin{abstract}
Resumo: O mamão é um fruto de grande importância econômica no Brasil, esta é amplamente cultivada para o consumo fresco e para uso em sucos, doces e geléias de frutas secas e cristalizadas, individuais ou com adição de outros produtos, como o coco. As pimentas do gênero Capsicum são especiarias picantes utilizadas no mundo inteiro. Aproximadamente um quarto da população mundial consome pimentas nas formas fresca ou processada. Quando utilizadas ainda frescas, conferem sabor e aroma a carnes, aves e peixes, são também utilizadas na forma de molhos e geleias. A elaboração de doces, em geral, é uma das formas empregadas para a conservação de frutas e agregar valor ao produto dos agricultores. O objetivo deste trabalho foi elaborar e avaliar sensorialmente o doce de mamão verde com coco e pimenta. O presente trabalho foi desenvolvido no laboratório de Biotecnologia Vegetal do Centro de Ciências Agrárias da Universidade Federal da Paraíba, Areia- PB. Foram preparadas duas amostras de doce de mamão verde com coco com e sem pimenta da espécie Capsicum annuum pertencentes ao Banco de Germoplasma do CCA / UFPB. Os tratamentos consistiram em duas formulações de doce de mamão com coco, sendo a formulação I (com pimenta) e a formulação II (sem pimenta) da espécie Capsicum annuum pertencentes ao Banco de Germoplasma do CCA / UFPB. Participaram da avaliação 60 julgadores não treinados que avaliaram a aceitação e intenção de consumo e as médias obtidas dos atributos das amostras avaliadas foram submetidas a estatística descritiva. Os testes utilizados na avaliação foram: escala hedônica e escala de atitude ou intenção. Conforme os resultados, a Formulação II doce de mamão com coco e pimenta apresentou preferência pelos julgadores, tanto para a análise sensorial como para intenção de consumo, demonstrando assim o potencial como produto para o mercado consumidor.
\end{abstract}

Palavras-chave: Capsicum annuum; desenvolvimento de um novo doce; intenção de consumo.

\section{Elaboration and sensory analysis of sweet green papaya with coconut and pepper}

\begin{abstract}
Papaya is a fruit of great economic importance in Brazil, it is widely cultivated for fresh consumption and for use in juices, candies and dried and candied fruit jellies, individually or with the addition of other products such as coconut. Peppers of the genus Capsicum are spicy spices used worldwide. About a quarter of the world's population consumes peppers in fresh or processed forms. When used fresh, they add flavor and aroma to meat, poultry and fish, and are also used in the form of sauces and jams. The making of sweets, in general, is one of the ways employed to preserve fruit and add value to farmers' produce. The objective of this work was to elaborate and sensorially evaluate the green papaya candy with coconut and pepper. The present work was developed in the Plant Biotechnology laboratory of the Center of Agricultural Sciences of the Federal University of Paraíba, Areia-PB. Two formulations of papaya candy with coconut samples were prepared, with formulation 1 corresponding to the sample without pepper and formulation 2 with addition of pepper of the species Capsicum annuum belonging to the CCA / UFPB Germplasm Bank. Sixty untrained judges participated in the evaluation and the averages obtained from the attributes of the evaluated samples were submitted to descriptive statistics. The tests used in the evaluation were: hedonic scale and attitude scale. According to the results, the formulation 2 of papaya with coconut and pepper was more preferred in the evaluation of judges, both for sensory analysis and for consumption intention, thus demonstrating the potential as a product to enter the consumer market.
\end{abstract}

Keywords: Capsicum annuum; developing a new candy; consumption intention.

\footnotetext{
${ }^{1}$ Submetido em 28/03/2019 e aprovado em 07/10/2019;

${ }^{2}$ Centro de Ciências Agrária, Universidade Federal da Paraíba, Areia, Paraíba, Brasil; E-mail: silvapriscilad@ @mail.com - ORCID: 0000-00026564-8428; nardielesouza01 @ gmail.com - ORCID: 0000-0003-3157-9679;

${ }^{3}$ Programa de Pós-Graduação em Agronomia, Universidade Federal da Paraíba, Areia, Paraíba, Brasil; E-mail: elizanilda@cca.ufpb.br ORCID: 0000-0001-7376-7569; angelapessoapb@gmail.com - ORCID: 0000-0002-7393-984X; manoelricardojunior@gmail.com - ORCID: 0000-0002-2074-7377; mailson@cca.ufpb.br - ORCID: 0000-0003-3096-6992.
}

Agropecuária Técnica, Areia-PB, v. 40, n. 3-4, p. 82-87, 2019 


\section{Introdução}

O mamão (Carica papaya L.) é um dos frutos de maior importância no Brasil, sendo a terceiroa mais consumido com grande relevância econômica e social, principalmente na geração de emprego e renda (Galo et al., 2014). Este fruto é amplamente cultivado para o consumo fresco e para uso em sucos, doces e geléias de frutas secas e cristalizadas (Rigotti, 2014). O mamão verde caracteriza-se pela baixa atividade antioxidante e vitamina $\mathrm{C}$, mas oferece um maior conteúdo de fibras que o fruto maduro (Mahattanatawee et al., 2006), sendo uma importante alternativa para diversificar a alimentação humana na forma de doces.

O coco oferece diversas formas de utilização, seja empregado para fins artesanais, alimentícios, nutricionais, medicinais e biotecnológicos (Carvalho, 2006). O fruto é também bastante usado fresco para uso culinário e também na produção de doces e bolos (Aragão, 2014).

As pimentas do gênero Capsicum são especiarias picantes utilizadas no mundo inteiro. Aproximadamente um quarto da população mundial consome este tipo pimentas nas formas frescas ou processada. Quando utilizadas ainda frescas, conferem sabor e aroma a carnes, aves e peixes, são também utilizadas na forma de molhos e geleias (Castro, 2014). Os produtos com base em pimentas incluem molhos, conservas, páprica, pimenta calabresa, frutos desidratados, geleias, pasta de pimenta, além de ser um ingrediente em diversos produtos alimentícios e ingrediente ativo na formulação de preparados farmacêuticos e cosméticos (Araújo et al., 2012; Rêgo et al., 2011).

Um dos fatores que aumentam o valor comercial da pimenteira é a pungência, considerada um dos maiores atrativos para os consumidores. Essa pungência é devida a vários alcaloides presentes nas pimenteiras, dentre eles a capsaicina e dihidrocapsaicina que são responsáveis por $90 \%$ de toda a pungência (Mansour-Gueddes et al., 2014).

A elaboração de doces, em geral, é uma das formas empregadas para a conservação de frutas, pois o efeito do teor de açúcar na atividade de água é muito significativo na conservação de alimentos. A diminuição da água livre é capaz de controlar os processos biológicos como multiplicação de microrganismos e agrega valor ao produto dos agricultores. Além disso, a temperatura empregada na elaboração de doces atua sinergicamente no controle dos microrganismos (Freitas et al., 2012).

Esses doces de mamão são preparados a partir de frutas devidamente selecionadas, este produto é feito com frutas inteiras ou em pedaços, sem sementes, com ou sem casca, e submetidos a cozimento em água, acondicionado em recipientes herméticos com calda de açúcar e submetido a tratamento térmico adequado (Durigan e Durigan, 2014) e já são bastante difundido no mercado consumidor, porém técnicas que conferem ao produto um diferencial a partir do incremento das formulações podem propiciar desenvolvimento de novo produto, agregando valor ao mesmo, sendo uma possível fonte de renda.

Ao desenvolver um novo produto, é imprescindível aperfeiçoar parâmetros, como forma, cor, aparência, odor, sabor, textura, consistência e a interação dos diferentes componentes, com o propósito de alcançar um equilíbrio integral e consequentemente uma boa qualidade e aceitabilidade do produto (Penna, 1999; Sapucay et al., 2009).

Devido à facilidade na obtenção dos ingredientes e preparo, os doces caseiros mostram-se como alternativa para $o$ desenvolvimento de novos produtos que poderão ser introduzidos no mercado com um apelo diferenciado, como também uma forma de minimizar o desperdício de alimentos e os efeitos sazonalidades da produção de matérias primas (Lima, 2010).

A análise sensorial é um campo muito importante na indústria de alimentos, pois contribui direta ou indiretamente para inúmeras atividades, como desenvolvimento de novos produtos, controle de qualidade, reformulação e redução de custos de produtos, relações entre condições de processo, ingredientes, e aspectos analíticos e sensoriais (Milagres et al., 2010). Deste modo, o objetivo deste trabalho foi elaborar e avaliar sensorialmente o doce de mamão verde com coco e pimenta.

\section{Material e Métodos}

O trabalho foi desenvolvido no Laboratório de Biotecnologia Vegetal do Centro de Ciências Agrárias da Universidade Federal da Paraíba, 
localizado no município de Areia- PB. Foram utilizadas duas amostras de doce de mamão verde com coco, sendo uma amostra com acréscimo de pimenta.

Os frutos de mamoeiro verde foram adquiridos no Centro de Ciências Agrárias e as pimentas vermelhas com baixa pungência utilizadas, pertencentes a espécie (Capsicum annиum), obtidas em casa de vegetação do banco de Germoplasma do CCA/UFPB. Para a amostra 1, utilizou-se $200 \mathrm{~g}$ de mamão formosa verde, $6 \mathrm{~g}$ de canela em pau, 5 cravos da índia, $400 \mathrm{~g}$ de açúcar, $120 \mathrm{~g}$ de coco ralado e $900 \mathrm{ml}$ de água. $\mathrm{Na}$ amostra 2 foram utilizados os mesmos ingredientes, porém com o acréscimo de 10 frutos de pimenta $(30 \mathrm{~g})$ no preparo do doce, seguindo a metodologia de Costa et al (2017) com modificações.

Inicialmente os frutos do mamoeiro foram higienizados com hipoclorito de sódio a $1 \%$ diluído em $1 \mathrm{~L}$ de água por 5 minutos, então realizou-se o enxague com água potável em abundância. Posteriormente, os frutos foram descascados, cortados em cubos pequenos e colocados em solução de bicarbonato de sódio com água, deixado em repouso por 2 horas. Após esse período foi feito o enxague dos frutos em água limpa. Em seguida, adicionou-se os frutos cortados em recipiente com o açúcar, coco ralado, canela em pau, cravos e água, sendo encaminhado para cozimento em fogo médio, $100^{\circ} \mathrm{C}$, por 45 minutos, para a amostra 2 houve o acréscimo da pimenta.

Quando os cubos do mamão apresentaram aspecto translucido, adicionou-se a calda previamente preparada com fogo baixo, $100^{\circ} \mathrm{C}$, até que houvesse a mistura total da calda com os mamões em cubo até apresentar mudança de cor, ficando com coloração marrom. Após a etapa anterior, as amostras do doce de mamão foram acondicionadas em recipientes de vidro devidamente esterilizado, fechado com tampa de metal e armazenado em refrigerador com temperatura de $6{ }^{\circ} \mathrm{C}$.

No preparo da calda do doce utilizou-se parte do açúcar definido para as formulações, sendo $200 \mathrm{~g}$ de açúcar cristal para $400 \mathrm{~mL}$ de água. Os ingredientes foram encaminhados ao fogo baixo até dissolver o açúcar e obtenção da calda, seguindo a metodologia de Neto \& Paiva (2006).
O teste da escala hedônica de aceitação global, expressa o grau de gostar ou de desgostar de um produto (ABNT, 1998). A escala utilizada neste teste foi a de 9 pontos, descrita em escala decrescente: (9) gostei extremamente; (8) gostei moderadamente; (7) gostei regularmente; (6) gostei ligeiramente; (5) não gostei, nem desgostei; (4) desgostei ligeiramente; (3) desgostei regularmente; (2) desgostei moderadamente e (1) desgostei extremamente (Tabela 1). O teste afetivo de escala de atitude, expressa a vontade do avaliador de consumir-um produto que lhe é oferecido, para esse teste utilizou-se a escala verbal de 7 pontos, onde em escala decrescente: (7) comeria sempre; (6) comeria muito frequentemente; (5) comeria frequentemente; (4) comeria ocasionalmente; (3) comeria raramente: (2) comeria muito raramente; (1) nunca comeria (Tabela 2).

Tabela 1 Ficha utilizada para avaliação da aceitação global do doce de mamão verde com e sem pimenta

\begin{tabular}{|c|c|c|}
\hline $\begin{array}{l}\text { Amostra: } \\
\text { Julgador } \\
\text { Data: }\end{array}$ & & \\
\hline \multicolumn{3}{|c|}{$\begin{array}{l}\text { Você está recebendo duas amostras codificadas. } \\
\text { Avalie globalmente cada uma segundo o grau de } \\
\text { gostar ou desgostar, utilizando a escala abaixo. }\end{array}$} \\
\hline (9) & Goste extremamente & ()$\left.^{\prime}\right)$ \\
\hline (8) & Gostei moderadamente & $($ ) \\
\hline (7) & Gostei regularmente & $($ ) \\
\hline (6) & Gostei ligeiramente & $($ ) \\
\hline (5) & Não gostei, nem desgostei & $($ ) \\
\hline (4) & Desgostei ligeiramente & \\
\hline (3) & Desgostei regularmente & $($ ) \\
\hline (2) & Desgostei moderadamente & $($ ) \\
\hline (1) & Desgostei extremamente & $($ ) \\
\hline
\end{tabular}

Fonte ABNT, NRB 14141, 1998.

A avaliação sensorial foi realizada em luz ambiente na Universidade Federal da Paraíba do Centro de Ciências Agrarias, Areia-PB com 60 julgadores não treinados situados na faixa etária de 18 a 45 anos do sexo masculino e feminino, onde foram orientados a realizar a lavagem da cavidade oral com água filtrada, no intervalo de cada amostra. Considerando a análise das duas amostras com avaliação dos mesmos parâmetros, aplicou-se questionários semiestruturados para as amostras avaliadas e os dados obtidos, sistematizados e analisados utilizando-se estatística descritiva. 
Tabela 2 Ficha utilizada na avaliação do doce de mamão verde com e sem pimenta

\begin{tabular}{l} 
Amostra: \\
Julgador \\
Data: \\
\hline Você está recebendo duas amostras codificadas. \\
Avalie globalmente cada uma segundo o grau de \\
intenção de consumo dos produtos, utilizando a \\
escala abaixo. \\
\hline (7) Comeria sempre muito \\
(6) Comeria \\
(5) Comeria frequentemente \\
(4) Comeria ocasionalmente \\
(3) Comeria raramente \\
(2) Comeria muito raramente \\
(1) Nunca comeria
\end{tabular}

Fonte ABNT, NRB 14141, 1998.

\section{Resultados e Discussão}

De acordo com os resultados obtidos na análise sensorial, observa-se que para a amostra 1 (doce de mamão sem pimenta), 28\% dos avaliadores atribuíram nota 9 (gostei extremamente) referente a escala de avaliação global, seguida da nota 8 (gostei moderadamente), correspondente a $14 \%$ dos entrevistados, sendo nota 3 (desgostei regularmente) a menor nota atribuída. Para amostra 2, houve maior aceitação em relação a amostra 1 , onde $45 \%$ dos entrevistados atribuíram nota 9 (gostei extremamente), seguida da nota 8 (gostei moderadamente), apresentando valor menor de acordo com 9\% dos avaliadores, indicando boa aceitação do doce de mamão com coco e pimenta, devido a pungência em relação a formulação sem acréscimo de pimenta (Figura 1).

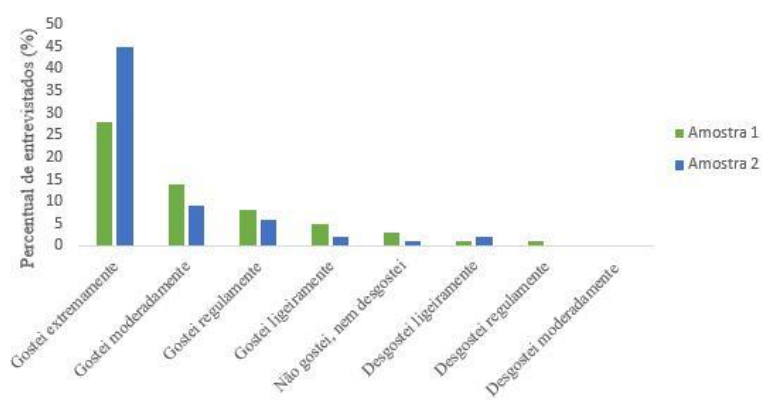

Figura 1 Aceitação global de doce de mamão verde com coco e pimenta Formulação 1: Doce de mamão verde com coco sem pimenta; Formulação 2: Doce de mamão verde com coco e pimenta.
Araújo et al. (2009), ao avaliarem sensorialmente formulações de geleias de pimenta com abacaxi, verificaram que a mesma obteve ótima avaliação dos julgadores, com 53\% atribuídos a nota 9 (gostei extremamente) para amostra com a presença de pimenta. Do mesmo modo, neste estudo a amostra do doce de mamão com coco e pimenta também foi melhor avaliada pelos julgadores.

Segundo Almeida et al. (2018) a análise sensorial identifica características essenciais no alimento, sendo por isso que a qualidade deste, está diretamente relacionada com a aceitação do consumidor. Geralmente aplicada no ramo alimentício nas etapas do desenvolvimento de novos produtos e controle de qualidade através de diversos testes, tais como os afetivos de preferência, aceitação e intenção de compra (Minin, 2010).

avaliação comercial do doce de mamão verde com coco e pimenta, observa-se que para a amostra 1, 23\% dos avaliadores atribuíram nota 7 (comeria sempre), sendo a mais citada entre as demais notas para intenção de compra, seguida da nota 6 (comeria muito frequentemente) com $13 \%$. A amostra 2 apresentou aceitação dos consumidores correspondente a $41 \%$ (comeria sempre), cerca de $13 \%$ dos julgadores expressaram que comeriam muito frequentemente e apenas $2 \%$ dos avaliadores demonstraram que nunca comeriam (Figura 2). Resultados semelhantes foram constatados por Castro et al. 2016, em estudo com geleias de frutas com pimenta, onde obteve boa aceitação em relação a intenção de consumo.

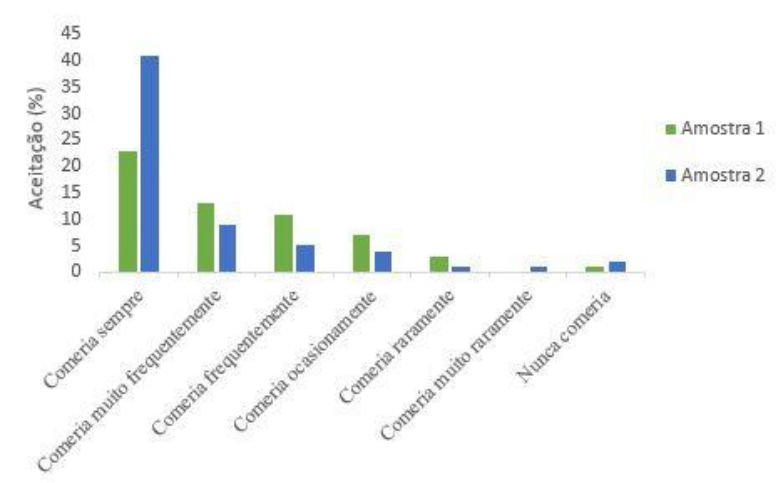

Figura 2 Análise sensorial de intenção de consumo de doce de mamão verde com coco e pimenta. Formulação 1: Doce de mamão verde com coco sem pimenta; Formulação 2: Doce de mamão verde com coco e pimenta.

Agropecuária Técnica, Areia-PB, v. 40, n. 3-4, p. 82-87, 2019

https://doi.org/10.25066/agrotec.v40i3-4.45166 
Diante dos resultados referentes as duas amostras, é possível observar que a amostra 2 cuja formulação apresenta acréscimo de pimenta foi melhor avaliada pelos julgadores, tanto para a avaliação global como para a intenção de consumo, sendo destacado pela pungência como atributo responsável por propiciar o diferencial ao doce. É notável que o doce de mamão verde com coco e pimenta torna-se um produto com potencial a entrar no mercado, devido a boa aceitação por parte dos avaliadores.

\section{Conclusão}

O doce de mamão verde com coco com acréscimo de pimenta obteve aceitação sensorial superior por parte dos julgadores em comparação o doce de mamão verde com coco tradicional, constituindo uma nova opção para introdução de um produto de sabor diferenciado no mercado. Este novo produto pode fornecer uma possível alternativa de emprego e renda, tendo em vista a praticidade no preparo e uso de produtos amplamente disponíveis.

\section{Referências}

ABNT. Associação Brasileira de Normas Técnicas. NBR 14141: escalas utilizadas em análise sensorial de alimentos e bebidas. Rio de Janeiro, 1998. 3p

Araújo, E. R.; Silva P.K.; Rêgo, E. R.; Bairral, M. A. A.; Santos, R. M. C.; Sapucay, M. J. L. C.; Farias, G. A.; Rêgo, M. M. Análise sensorial e de aceitação comercial de geléia de pimenta com acerola. Horticultura Brasileira, v. 27, n. 2, p. S1545-S1550, 2009.

Araújo, E. R.; Rêgo, E. R.; Sapucay, M. J. C.; Rêgo, M. M.; Santos, R. M. C. Elaboração e análise sensorial de geleia de pimenta com abacaxi. Revista Brasileira de Produtos Agroindustriais, v. 14, n. 3, p. 233-238, 2012.

Aragão, W. M. Costa, N. F. E.; Ribeiro E. F. 2014.O potencial do coqueiro híbrido para cocoicultura brasileira. Disponível em:< http://www.agencia.cnptia.embrapa.br/gestor/coco/arvore /CONT000g15m652b02wx5ok0xkgyq5dmanf8w.html

>Acesso em: Jun. 2018.

Carvalho, J. M.; Maia, G. A.; Sousa, M. H. P.; Maia, G. A. J. Água de coco: propriedades nutricionais, funcionais e processamento. Semina: Ciências Agrárias, v. 27, n. 3, p. 437-
452, 2006. http://dx.doi.org/10.5433/16790359.2006v27n3p437

Castro, G.; Lopes, H. A.; Silva, D. A. T. P.; Gorayeb, T. C. C. Elaboração de geleia de frutas com pimenta dedo de moça (Capsicum baccatum var. Pendulum) Revista do Agronegócio - Reagro, v. 5, n. esp., p. 45-57, 2016.

Costa, D. S, P, M.; Rêgo, R. E.; Silva, F. E. K.; Rolim, R. R.; Rêgo, M. M.; Sousa, S. E. Análise sensorial de trufas de maracujá com pimenta. Anais do III Encontro Nacional da Agroindústria: desafios da segurança alimentar.1 ed. Bananeiras- PB, 2017. 123p.

Durigan, B. F. M.; Durigan, F. J. Tecnologia Póscolheita e Processamento de Mamão: Qualidade e Renda aos Produtores Roraimenses. Boa Vista: Embrapa, 1981. 17p.

Freitas, M. L. F.; Menezes,C. C.; Carneiro, J. D. S.; Reis, R. P. Diagnóstico do consumo e processo produtivo de doces de frutas produzidos artesanalmente. Brazilian Journal of Food \& Nutrition/Alimentos e Nutrição, v. 23, n. 4, p. 589-595, 2012.

Galo, J. Q. B.; Souza, M. L.; Kusdra, J. F.; Mattiuz, C. F. M. Conservação pós-colheita de mamão 'sunrise solo' com uso de quitosana. Revista Brasileira de Fruticultura, v. 36, n. 2, p. 305-312, 2014. http://dx.doi.org/10.1590/01002945-194/13

Lima, M. I. P. Dicas especiais de aproveitamentos de alimentos. Prefeitura Municipal de Londrina/ Secretaria Municipal da Agricultura e Abastecimento. Londrina: PML/SMA, 2010. 12p.

Mahattanatawee, K.; Manthey, J. A.; Luzio, G.; Talcott, S. T.; Goodner, K.; Baldwin, E. A. Total antioxidant activity and fiber content of select Florida - grown tropical fruits. Journal of agricultural and food chemistry, v. 54, n. 19, p. 7355-7363, 2006. https://doi.org/10.1021/jf060566s

Milagres, P. M.; Dias, G.; Magalhães, A. M.; Silva, O, M.; Ramos, M. A. Análise físicoquímica e sensorial de doce de leite produzido sem adição de sacarose. Revista Ceres, v. 57, n.4, p. 439-445, 2010. http://dx.doi.org/10.1590/S0034-737X2010000400001 
Minin, V. P. R. Análise sensorial: estudos com consumidores. $2^{\mathrm{a}}$ ed. Viçosa: Editora UFV, 2010.

Neto, S. R. M.; Paiva, A. F. F. Doce de frutas em calda. $1^{a}$ ed. Brasília: Embrapa Informação Tecnológica, 2006. 22p.

Penna E. W. Desarrolho de alimentos para regimenes especiales. In: Morales, R.H; Tudesca, M.V. Optimizacion de formulaciones. Santa Curz de la Sierra, Bolivia, 1999.

Rêgo, E.R.; Finger, F.L.; Nascimento, N.F.; Araújo, E.R.; Sapucay, M.J.L.C. Genética e melhoramento de pimenteiras Capsicum spp. In: Rêgo, E.R.; Finger, F.L.; Rêgo, M.M. (Org.). Produção, genética e melhoramento de pimentas (Capsicum spp.). Imprima, Recife, 2011. p. 117-136.

Rigotti, M. Cultura do Mamoeiro -Disponível em:

http://www.portaldahorticultura.xpg.com.br/CulturadoMa moeiro.pdf >. Acesso em: 10 jun. 2014.

Sapucay et al. Elaboração e análise sensorial de geleia de pimenta com abacaxi. Horticultura brasileira, v. 27, n. 2 (Suplemento - CD Rom), ago. 2009. 